\title{
Paper C-V2X Communications for the Support of a Green Light Optimized Speed Advisory (GLOSA) Use Case
}

\author{
Ioannis P. Chochliouros ${ }^{1}$, Anastasia S. Spiliopoulou ${ }^{1}$, Alexandros Kostopoulos ${ }^{1}$, George Agapiou ${ }^{1}$, \\ Pavlos Lazaridis ${ }^{2}$, Zaharias Zaharis ${ }^{3}$, Tao $\mathrm{Chen}^{4}$, Athanassios Dardamanis ${ }^{5}$, Michail-Alexandros \\ Kourtis $^{6}$, Marinos Agapiou ${ }^{7}$, Uwe Herzog ${ }^{8}$, and Latif Ladid ${ }^{9}$ \\ ${ }^{1}$ Hellenic Telecommunications Organization, Athens, Greece \\ ${ }^{2}$ University of Huddersfield, Huddersfield, United Kingdom ${ }^{3}$ Aristotle University of Thessaloniki, Thessaloniki, Greece \\ ${ }^{4}$ VTT Technical Research Centre of Finland, Espoo, Finland ${ }^{5}$ SmartNet, Athens, Greece \\ ${ }^{6}$ ORION Innovations Private Company, Athens, Greece ${ }^{7}$ Independent Consultant, Athens, Greece \\ ${ }^{8}$ Eurescom, Germany ${ }^{9}$ University of Luxembourg/SnT, Luxembourg
}

https://doi.org/10.26636/jtit.2021.152321

\begin{abstract}
Rapid expansion of $5 \mathrm{G}$ affects a number of sectors, including vehicular communications relying on cooperative intelligent transportation systems (C-ITS). More specifically, in the context of the Internet of Vehicles (IoV), a particular emphasis is placed on modern cellular V2X (C-V2X) technologies aiming to further improve road safety. This work originates from the detailed scope of the ongoing 5G-DRIVE research project promoting cooperation between the $\mathrm{EU}$ and China, with the aim of demonstrating IoV services that rely on vehicle-to-infrastructure (V2I) communications. With the C-V2X approach serving as a point of departure, we analyze and describe a specific green light-optimized speed advisory (GLOSA) use case, for which we provide a detailed descriptive framework, a proposed architectural framework for trials, as well as specific KPIs for the joint assessment of trials between the EU and China. We also discuss the context for performance test procedures to be conducted as part of the intended trials. GLOSA provides end-users with short-term information on upcoming traffic light status to optimize traffic flows, help prevent speed limit violations, improve fuel efficiency, and reduce pollution.
\end{abstract}

Keywords-5G, cellular V2X, cooperative awareness messages, cooperative intersection, cooperative ITS, green light-optimized speed advisory, intelligent transportation systems, traffic efficiency, vehicle-to-everything.

\section{Introduction}

\subsection{G as an Enabler for Modern ITS}

The 5G technology used in mobile and wireless communications fosters the evolution of modern societies and economies. This strongly affects the way in which mobile communications and enormous amounts of data generated by various applications may transform our modern way of living. 5G supports connectivity requirements of a num- ber of heterogeneous infrastructures, thus facilitating the use of convenient solutions designed by various market actors. This means that $5 \mathrm{G}$ contributes to the creation and operation of a new form of underlying network infrastructures offering increased agility and adaptability levels, and effectively supporting a variety of technologies. This, in turn, fosters development and economic growth, as $5 \mathrm{G}$ becomes capable of promoting innovative solutions that may be beneficial for the participating end-users (i.e. residents, corporations, state authorities, and many others) and, therefore, of improving our living standards.

In particular, as $5 \mathrm{G}$ promotes also the inclusion of cognitive and automation features, as well as the latest advances in artificial intelligence (AI), platforms are established and operated that support the design and the offering of new services, such as mission-critical (MC) communications and services based on the Internet of Things (IoT). Such facilities offer enhanced capacity and performance for all communication methods, serving various sectors [1]. With that taken into consideration and in view of the wide-scale and/or global digital transformation taking place, unique opportunities may be offered by offering new processes and unprecedented opportunities for the benefit of the industrial sector [2]. $5 \mathrm{G}$ is not a simple transition to a next generation of mobile communications. Instead, it becomes a true enabler of progress, increasing the level of performance, reducing latency and boosting network/service reliability, even for MC applications. Furthermore, 5G is also a promoter of market growth, as it supports a great number of the so-called vertical industries that will play an important part in the new reality and will become active participants of the new digital era [3].

Introduction and inclusion of cooperative intelligent transportation systems (ITS) [4] is expected to play an important role in this global evolutionary process. ITS are perceived 
to be a sector that has been evoking the largest amount of interest recently, attracting significant national and international investments aiming to promote novel solutions. As ITS are explicitly correlated with every-day activities, policy makers, the automotive industry, telecom providers, transportation experts and other "actors" have shown significant interest in that particular area. The aim of their actions has been to offer a portfolio of new services and, most importantly, to deal with existing obstacles, such as traffic congestion, road safety, transport efficiency and environmental conservation. All this means that further growth has been thwarted. ITS may be considered as effectively including all types of transportation infrastructures, such as streets, highways, bridges, tunnels, railways, ports and airports, as well as vehicles (not only cars, but also buses, lorries, trains, aircraft and waterborne vessels).

The fact that ITS affect the majority of various modes of transport, simultaneously fostering multi-modality, is an important factor too. The range of the services offered on the market is immense and depends on the enabling technologies applicable that are relevant for the specific cases. ITS may include, for example, management enhancing services, such as those related to navigation, traffic signal control systems, container management systems, variable message signs and enforcement systems for monitoring applications (e.g. CCTV systems). However, the notion may also be extended to cover more advanced applications that integrate live data from other sources (such as parking guidance and information systems or weather information systems), working in more complex environments and being able to correlate data from various sources.

\subsection{Challenges for Cooperative ITS in Vehicular Applications}

Applications for connected vehicles, in a broad sense of the word, are an area that is attracting strong interest due to the potential progress, especially when the support offered by 5G-based innovative features is taken into consideration. This calls for a new strategic approach that will address previous concerns and/or measures, such as those applied by ETSI [5], as well as those developed occasionally in Europe and at other locations around the world. ITS support effective, combined and inclusive use of communication, computer and control tools to meet stricter system performance-related and operational requirements. This approach also takes into account additional requirements established in order to satisfy other priorities, such as environmental concerns, reduced energy consumption, better performance and level of service compliant with applicable criteria, enhanced security even in complex operational environments, and mobility, just to mention a few.

"G5" - not to be confused with $5 \mathrm{G}$, is such a reliable standard for car-to-car communications. It is based on the evolution of the Wi-Fi standard (i.e. IEEE 802.11.p), being one of the iterations of the original IEEE 802.11 solution, aiming to provide wireless access in vehicular environments. G5 relies on data exchanged between vehicles moving at high speeds, as well as between vehicles and roadside infrastructure. The process is known as V2X communication and relies on the ITS-licensed band of $5.9 \mathrm{GHz}$ $(5.85-5.925 \mathrm{GHz})$. With the benefits offered by $5 \mathrm{G}$ taken into consideration and in order to ensure enhanced functionalities, the development of new services, occasionally based on the hybrid communication approach, should be expected.

Modern communications technologies and the related invehicle and on-the-road infrastructures have all brought about several advantages for drivers, the automotive industry as such and for other stakeholders, especially in the transportation and the emergency services ecosystems, providing better driving conditions and promoting higher security levels. Recently, research efforts have been focusing on cooperative ITS (C-ITS), where vehicles communicate with each other and/or with the infrastructure [6]. Thus, C-ITS goes a step further by intending to improve the quality and to increase the reliability of information available, such as data about the vehicles, their location and the environment in which they move. This results not only in improving the existing applications relied upon by road users, but also in developing new ones, thus leading to better efficiency of transport and to increased safety [7]. As a critical policy challenge for modern societies is to reduce the number of road accidents and to advance road safety and security, it is essential for vehicles to detect what is happening around them, predict what may potentially occur next and take both protective and proactive measures, accordingly.

\subsection{Cellular V2X Communications Supporting C-ITS}

In a broader context, we could consider V2X communication as being a variety of a wireless sensor-based system, allowing vehicles to share and exchange information with each other and with other parts of the infrastructure via dedicated communication channels. When attempting to compare this solution with "standard" sensors (such as radar, LIDAR, lasers, ultrasonic detectors, etc.) one needs to mention that the utilization of a V2X system offers extra benefits, as such an approach enable to obtain out-of-sight information, to test hidden threats and to expanding the driver's perception. In consequence, driving safety and efficiency are improved, and driving comfort is boosted by various driving automation systems [8].

The vehicle-to-everything (V2X) technology is the next big feature to evolve further the automotive and transportation industry [9]. Through various communication technologies, V2X allows a vehicle linking to other vehicles, pedestrians, road infrastructure, the Internet, and other entities in the transportation ecosystem. V2X also provides the infotainment experiences, and eventually supports transition towards thing in terms of autonomous driving. In [10], the authors forecast that, by the year 2022, there will be more than 125 million vehicles connected by means of various V2X technologies, offering an unprecedented level of safety, expanding the range of known transportation services and offering other novel advantages. Thus, it becomes 
obvious that it is now the critical phase to test and deploy V2X technologies and infrastructures, especially by combining those processes with the deployment and testing of modern $5 \mathrm{G}$ infrastructures.

In recent years, different regions in the world have conducted intense V2X trials. Two distinct V2X technical paths are followed by the automotive industry. ETSI ITSG5, an approach based on 802.11p technologies [11], is one of them. 3GPP LTE-V2X [12] that is rooted in 3GPP standards is the other. Different regions have their own preferences concerning the technologies. China opts for cellular V2X (C-V2X or LTE-V2X) as its national standard. In Europe, the debate is ongoing on how to adopt the technologies. The 5G Automotive Association (5GAA), the international association with the mission to promote $\mathrm{C}-\mathrm{V} 2 \mathrm{X}$ technologies, expects that the first commercial deployments of V2X will occur in China and Europe, while deployments in the US and other parts of Asia will follow closely. Considering the fact that the life cycle of road infrastructure equals usually 30 years, and that the life cycle of a car is $10-15$ years, selection of V2X will be critical for the future evolution of technologies.

Due to compatibility reasons, it is crucial that the various regions cooperate to ensure harmonization of technologies. With this kept in mind, Europe and China have established cooperation on validation of the C-V2X technology through joint research and trials. Car manufacturers, road authorities, telecom vendors, and mobile operators team up to test key C-V2X technologies and use cases. The 5G-DRIVE EU-funded project [13] is one of the undertakings concerned with working on C-V2X trials with China. The aim is to compare the performance in joint V2X use cases, and to identify any potential interoperability problems.

The work is organized as follows. Section 1 serves as an introduction. Firstly, it identifies the important role of $5 \mathrm{G}$ for the promotion of modern ITS, then it focuses on the challenges for cooperative ITS in vehicular applications. Lastly, it discusses modern cellular V2X communications supporting the automotive industry in the $5 \mathrm{G}$ era. Section 2 presents a wider scope of the 5G-DRIVE project, aiming to promote trials and tests between the EU and China for two scenarios, one of which is relevant to V2X communications, especially those relying on C-V2X technologies. Then, Section 3 presents a more detailed framework for C-V2X communications, initially by discussing recent evolutionary options stemming from $5 \mathrm{G}$ growth, and then by introducing the selected green light optimized speed advisory (GLOSA) use case. More specifically, we assess the innovative features of this important use case which offers major benefits for the market and we propose a dedicated novel architecture within the 5G-DRIVE-specific context, based on $\mathrm{C}-\mathrm{V} 2 \mathrm{X}$, being one of the novelties introduced by this work to international literature. Then, we propose several KPIs for the assessment of trials and the scope of the intended performance test procedures. Section 4 completes the work by offering general overview of the scheduled trials intended to focus upon compliance tests performed in accordance with the ETSI EN 302571 standard. Based on some early findings, we also assess trials performed in the EU and China.

\section{5G-DRIVE Project Framework}

\subsection{Trial and Experiment Sites in the EU}

The 5G-DRIVE project [13] is an active element of the well-established H2020 ICT-22-2018 Call (EU China 5G Collaboration) scheme, aiming to ensure collaboration between the EU and China in order to work out solutions guaranteeing effective use of $5 \mathrm{G}$ technologies and to handle spectrum-related challenges before the expected wide scale roll-out of 5G. Figure 1 shows the fundamental 5G-DRIVE concept that focuses on the three illustrated core streams. Figure 1 depicts also the expected conceptual flow: from research, to adaptation to existing test-beds, to commercial test-bed deployments, to real-world trials of $5 \mathrm{G}$ radio access networks (RAN) and of the target 5G networks. 5G-DRIVE supports collaboration between solid research competence, commercial grade test-beds and some of the stakeholders who will eventually become major customers of the $5 \mathrm{G}$ systems and the proposed applications.

The 5G-DRIVE project incorporates extensive 5G testbed installations provided by three research organizations - members of the consortium: the University of Surrey (UoS), the VTT Technical Research Centre of Finland (VTT), and the Joint Research Centre of the European Commission (JRC) [14]-[15]. While all three test-beds are equipped with commercial grade hardware to ensure reliability of the obtained results, each one has been arranged to serve a dedicated purpose. The Surrey test-bed supports capacity provision in very dense deployments over a $4 \mathrm{~km}^{2}$ area. The VTT's Espoo test-bed validates the use of slicing and V2X. The JRC facility in Ispra, Italy, allows the testing of new V2X technologies in any part of the network in a fully-controlled environment. All test-beds allow for gradual introduction and testing of new equipment, as well as for the inclusion of new mechanisms, algorithms and protocols. In the research stream, the project investigates network and RAN slicing, mobile edge computing (MEC), massive multiple-input multiple output (MIMO) for 5G New Radio (NR) [16], as well as software-defined network (SDN) and network function virtualization (NFV) techniques applied to different traffic and load scenarios. The techniques and mechanisms relied upon in the research stream are currently under development and will be used in the most appropriate test-bed, with the final aim of deploying them in all three test-beds, if possible.

The project's experimental test-beds/facilities intend to satisfy several exact prerequisites [17], including the following:

- they need to offer an adequate level of flexibility, thus allowing for the inclusion - and use - of various characteristics of the involved technologies (distinct physical layers, frequency bands, etc.) at the differ- 


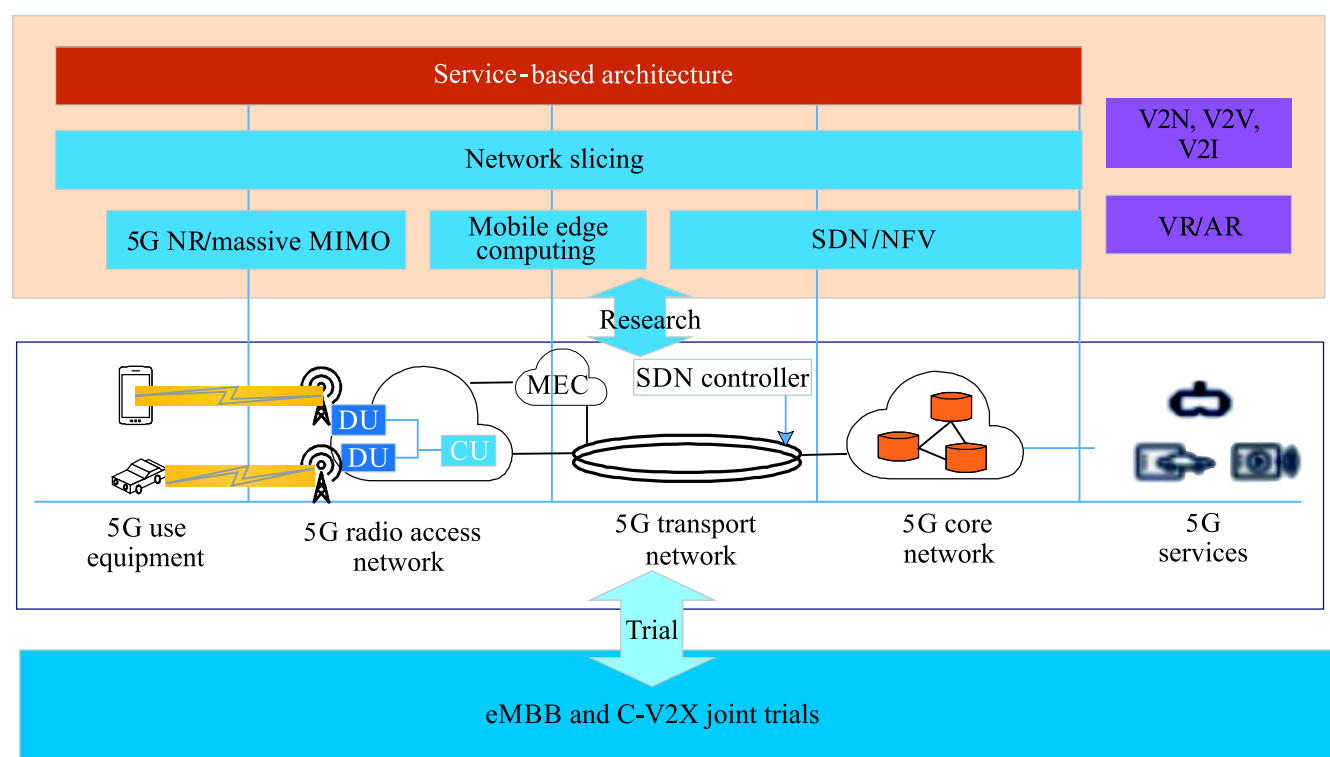

Fig. 1. Overall concept of the 5G-DRIVE project.

ent layers and in the related network components, on a case-specific basis,

- they need to be easily reconfigurable, thus allowing for dynamic adaptation to any specific testing requests made by the experiment participants,

- they need to support open-source solutions, so that their potential may be expanded by relying on the competence of the broadest possible scientific community,

- they need to offer testable and repeatable results, where necessary, to guarantee fair testing and reasonable comparison of the distinct technologies,

- they need to be complete in order to enable incorporation of all potential elements of a modern 5G ecosystem, such as mobile network operators (MNOs), virtual operators, end-users, machine-tomachine (M2M) applications, and the IoT,

- they need to support heterogeneity of the radio and optical interfaces tested, but also of specific contexts, including body-centric communications, vehicular networks, advanced robotics, etc.,

- they need to be site-agnostic, to the extent possible, with the purpose of testing technologies and the related facilities in dissimilar backgrounds,

- they need to be topology-agnostic, with the aim of supporting all potential wireless solutions, including cellular and satellite technologies, and topologies ranging from small cells to macro-cells.

\subsection{Aims and Objectives of the 5G-DRIVE Effort}

The main goal of 5G-DRIVE is to conduct 5G trials focusing on two dedicated scenarios:

- the first one is related to enhanced mobile broadband (eMBB) $(3.5 \mathrm{GHz})$ - a priority band in the two regions for early introduction of very high-rate services. It is used for rendering typical mobile broadband services, as well as augmented reality (AR) services and is supported by the Surrey and Espoo trial sites,

- the other scenario is related to LTE-V2X-based Internet of Vehicles (IoV) using the $5.9 \mathrm{GHz}$ band for vehicle-to-vehicle and the $3.5 \mathrm{GHz}$ band for vehicleto-network applications. The demonstrations will rely on real-life setups. Corresponding trials are conducted at Espoo and JRC sites.

The overarching aim of the project is to establish reliable collaboration and interactivity between recent $5 \mathrm{G}$ developments in the European Union and China, through joint trials and research activities in order to facilitate technology convergence, spectrum harmonization and business innovation, before any probable large-scale market efforts related to $5 \mathrm{G}$ deployment are undertaken. The expectation is to define requirements for the joint provision of corresponding services by the consortium members, especially with the participation of mobile operators and other stakeholders from the automotive and intelligent transports markets. The collaborative assessment allows to select the use cases, define trial requirements and to perform subsequent implementation and analysis. The inclusion of variable stakeholders is important to ensure that the trials and solutions meet the requirements of different vertical domains.

The 5G-DRIVE effort is based on existing 5G norms and on those that are currently under development, namely on 
releases 13-14 of the 3GPP standard. Some issues covered by future releases have been taken into account as well, where possible. The project relies on testing opportunities offered by the three existing $5 \mathrm{G}$ test-beds provided by the participating partners, all aiming to test and validate innovative applications and related services. For this purpose, the project has developed key $5 \mathrm{G}$ technologies and pre-commercial test-beds for eMBB and V2X services in collaboration with a twinned Chinese project led by China Mobile, intending to compare the implementation methodologies and results. Trials for testing and validating key $5 \mathrm{G}$ functionalities, services and network planning solutions are actually taking place in eight cities across the EU and China. The main targets of this close collaboration scheme are illustrated in Fig. 2.

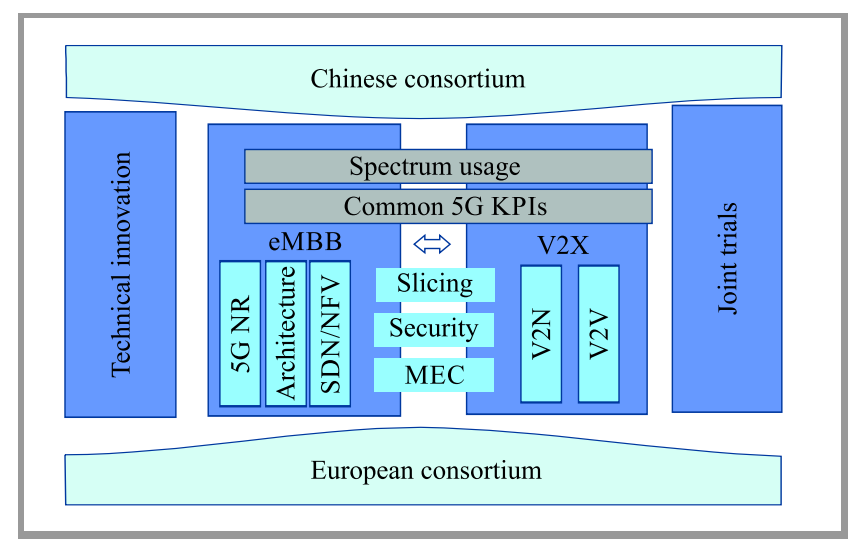

Fig. 2. EU-China 5G-DRIVE collaboration.

\section{Scope for Collaborative V2X Communication}

\subsection{General Framework}

Vehicle-to-Everything (V2X) communications were conceived as a means of transferring information from a vehicle to any entity that may have an impact on the vehicle, and vice versa. It is a vehicular communication system that integrates other more specific types of communications, such as vehicle-to-infrastructure (V2I), vehicleto-network (V2N), vehicle-to-vehicle (V2V), vehicle-topedestrian (V2P), vehicle-to-device (V2D) and vehicle-togrid (V2G) solutions. Thus, V2X may be perceived as a wireless technology enabling the exchange of data between a vehicle and its surroundings, whatever the latter may be. The core motivations behind $\mathrm{V} 2 \mathrm{X}$ are to increase road safety, boost traffic efficiency and generate energy savings. The process of sharing, speed and position data between a given vehicle and the surrounding vehicles and/or infrastructures may enhance the driver's awareness of potential dangers and may significantly increase their ability to avoid collisions, resulting in a reduced number fatalities and in lower severity of injuries [18]. Fluidity of traffic may be increased as well by providing warnings about imminent traffic congestions and by suggesting alternative routes. Eco-friendly driving styles may be encouraged as well, reducing $\mathrm{CO} 2$ emissions by adaptive cruise control solutions and by relying on smarter transportation management systems.

Two types of existing V2X communication systems may be distinguished, according to their underlying technology: they may be WLAN-based or cellular-based.

IEEE first published the specification of WLAN-based V2X (IEEE 802.11p) in 2012 [19]. 802.11p is an extension of the $802.11 \mathrm{a}(\mathrm{Wi}-\mathrm{Fi})$ standard that was defined by IEEE in 2009. In 2012, 802.11p was included in the overall IEEE 802.11 standard, but the informal term of $802.11 \mathrm{p}$ still continues to be used. The 802.11 p-based multiple access mechanism (carrier sense multiple access protocol with collision avoidance, CSMA-CA) is a statistical protocol for establishing direct communications, as well as for V2V and V2I connections [20]. Primary V2X communication relies on the WLAN technology and is established directly between vehicles that make up the so-called vehicular ad-hoc network, providing that V2X senders are located within each other's range. Therefore, no infrastructure is required for the vehicles to communicate, which is of fundamental importance to guarantee safety in remote areas. WLAN is suitable for V2X communications as it is a low latency solution. It transmits several messages that are known as cooperative awareness messages (CAM), decentralized environmental notification messages (DENM) or basic safety message (BSM) [21]. The amount of data contained in these messages is very low. The radio technology used relies on the WLAN IEEE 802.11 family of standards and is known in the US as wireless access in vehicular environments (WAVE), and in Europe as ITS-G5. 3GPP initiated early standardization of cellular V2X (C$\mathrm{V} 2 \mathrm{X}$ ) as part of release 14 in 2014 [22]. The applicable specifications were published in 2016. Because C-V2X relies on LTE as its underlying technology, it is often referred to as C-V2X to differentiate it from the 802.11 p-based V2X technology. The functionalities it supports include direct communication (V2V, V2I) and wide area cellular network communication $(\mathrm{V} 2 \mathrm{~N})$. In release $15,3 \mathrm{GPP}$ continued the process of standardizing C-V2X based on 5G [23]. Therefore, to indicate the underlying technology, the term of new radio $\mathrm{V} 2 \mathrm{X}(\mathrm{NR}-\mathrm{V} 2 \mathrm{X})$ is often used in contrast to LTE-based V2X (LTE-V2X). In either case, C-V2X is a generic term used to refer to cellular-based V2X communications, irrespective of the specific underlying type of mobile communication technologies used (4G or $5 \mathrm{G}$ ). In release $16,3 \mathrm{GPP}$ has further enhanced C-V2X functionality [24], and work in this regard is still in progress. 3GPP's main goal is to ensure enhanced data rates and to offer advanced ITS services. In other words, it strives to achieve objectives considered to be the key part of the future vehicular telecommunications landscape.

\subsection{GLOSA Use Case - Conceptual Description}

As far as the 5G-DRIVE V2X use cases are concerned, GLOSA and intelligent intersection are two solutions that are currently in their deployment and continuous assess- 
ment phase. European trials use the lab and the stable communication environment available in Ispra, Italy and the dedicated ITS back-office services tailored for the needs of the Espoo, Finland trial site. Mobile-edge computing and virtual traffic light technologies are implemented as well for checking LTE-based communication channels and C-V2X user equipment (UE) nodes.

The architecture of the system follows the European mobile edge computing (MEC) guidelines [25]-[26] and is aligned with the C-ITS DEMN message format [27]. Even if the devices are different between Europe and China, the results are intended to be comparable, as the trials rely on a common set of KPIs.

Extreme fuel consumption experience in urban settings is closely associated with driving in heavy traffic that is also characterized by larger speed fluctuations and frequent stops at intersections. An improvement in the flow of city traffic allows to lower fuel consumption and $\mathrm{CO} 2$ emissions by reducing the number of stops, shortening delays and maintaining moderate speeds throughout the entire journey. One way to eliminate the unnecessary stop-and-go driving in cities is to optimize traffic signal timing in a dynamic manner, by adapting it to the current traffic situation.

Historically, signal timing optimization tools were developed to reduce delays and stops experienced by urban drivers. In recent years, new methods in traffic signal optimization have integrated the changes in the drivers' behavior in order to achieve the highest level of performance at intersections with traffic lights [28]. Today, vehicles can be equipped with a variety of sensors, driver assistance tools and safety related systems. Integration of cellular communication systems with cars/vehicles has resulted in an improvement of both safety and comfort levels. The solutions are now used in millions of cars and a trend showing that continued rapid growth may be expected is evident. In fact, many of the use cases, previously described in the respective ETSI ITS specifications and/or other documents [5]-[6], are now a reality thanks to existing cellular network connections. Cellular networks allow a variety of warnings to be sent to cars. These include, for instance, warnings about slow or stationary vehicles, road work warnings, weather condition warnings, hazard warnings, in-vehicle signage and speed-limits. Connected vehicles are capable of establishing two-way wireless communications (of the V2V and V2I variety) that may be effectively used for a variety of mobility and safety-related applications. One such application is known as GLOSA [29]. The system uses accurate information about traffic signal timing and locations to guide drivers, relying on I2V communications to offer speed recommendations that make the commute smoother, reducing stopping and allowing to smoothly pass through traffic signals [30]. A GLOSA implementation may be evaluated for two types of traffic signal timing schemes: predictable fixed-time signal timing and unpredictable actuated-coordinated signal timing.

GLOSA systems have been shown to be able to reduce both $\mathrm{CO} 2$ emissions and fuel consumption [31] by providing drivers with speed recommendations when approaching a traffic light. For the system to reach its maximum potential, it is essential to appropriately forecast all different types of traffic light configurations, including also adaptive systems in which signals may change with lead times as short as $1 \mathrm{~s}$. The GLOSA application offers the benefit of timely and accurate information about traffic light cycles and locations via I2V communication. In consequence, drivers are provided with speed advice, ensuring that more constant speeds may be maintained and that less time is spent while the vehicle is stopped at traffic lights [32]. A reliable solution requires that traffic intensity, communications between traffic lights and vehicles, as well as driver behaviors be modeled in an effective manner. Research has been performed in each one of the aforementioned areas, but comprehensive simulations taking into account the dynamics of all parameters are scarce in the international literature.

The main goal of the GLOSA service is to predict the green phases of traffic lights and to provide drivers with reliable information enabling them to pass traffic lights during the green light phase. The green-wave assistant and the deceleration assistant are the two key applications of the system. The C-Roads Platform [33], being a joint initiative of the European Union Member States and road operators, established for testing and implementing C-ITS services in light of cross-border harmonization and interoperability, offers two methods of integrating the GLOSA service with vehicles: via ETSI G5, directly with the on-board computer of the vehicle, and via the mobile network, with a dedicated smartphone application. The green wave assistant shows information allowing the driver to reach the green phase at the next signal-controlled junction. Consequently, unnecessary stopping and acceleration may be prevented. The deceleration assistant works by informing the driver that he/she cannot reach the green phase at the next signalcontrolled junction. With the help of these two applications, drivers may adapt their driving behaviors based on the information sent and received by the vehicle, therefore boosting efficiency and comfort levels. The GLOSA application offers the advantage of providing timely and accurate information about traffic light cycles and positions through I2V communication. In consequence, drivers are provided with speed advice, ensuring that more constant speeds may be maintained and that less time is spent while the vehicle is stopped at traffic lights.

\subsection{Proposed Architecture}

GLOSA is a Day-1 signage C-ITS service [34] aiming to inform end-users (i.e. drivers) about the speed that needs to be sustained, within the applicable legal limits, to approach an upcoming traffic light during the green phase. Although the individual user interface (UI) features are manufacturer specific, GLOSA notifications provided to end-users usually follow the structure below:

- the upcoming traffic light phase will change in $\mathrm{x}$ seconds (countdown, with $\mathrm{x}$ usually varying between 10 and $20 \mathrm{~s})$, 
- maintain $35 \mathrm{~km} / \mathrm{h}$ - an optimal value to reach the upcoming traffic light during the green phase, in line with the applicable legal speed limits prevailing in a given,

- the driver will reach the upcoming traffic light during the red phase.

GLOSA provides drivers of approaching vehicles with short-term information on the status upcoming traffic lights, thus optimizing traffic flows and helping prevent speed limit violations, improving fuel efficiency and reducing pollution [35]. The system requires that intersection topology be determined, including geographical coordinates of entry and exit lanes that are transmitted in the form of a V2X I2V MAP message. This information is used by the receiving vehicles to calculate the relevance of the received notifications, taking into account their position relative to a given traffic light. The MAP message [36] is the basic requirement in many use cases, including GLOSA, as it provides a geographic reference for other messages. Not all vehicles have the same map installed on board and some of them may even have no map at all. Therefore, the MAP message is a platform that serves as an independent point of reference for all stations being a part of the ITS.

In Fig. 3, the architecture proposed for the test phase is presented. The key components of this particular use case are:

- A physical/virtual traffic light along with its controller to orchestrate the transitions between the red, amber and green phases. For the purpose of the experiment evaluating this particular use case, the traffic light may be either of the physical (i.e. a commercial, end-user product) or a virtual variety (software running on / communicating with the roadside unit (RSU) and performing the transitions ordered by the controller);

- An $L T E-V 2 X R S U$ installed at the traffic light location (if physical) or running on / communicating with

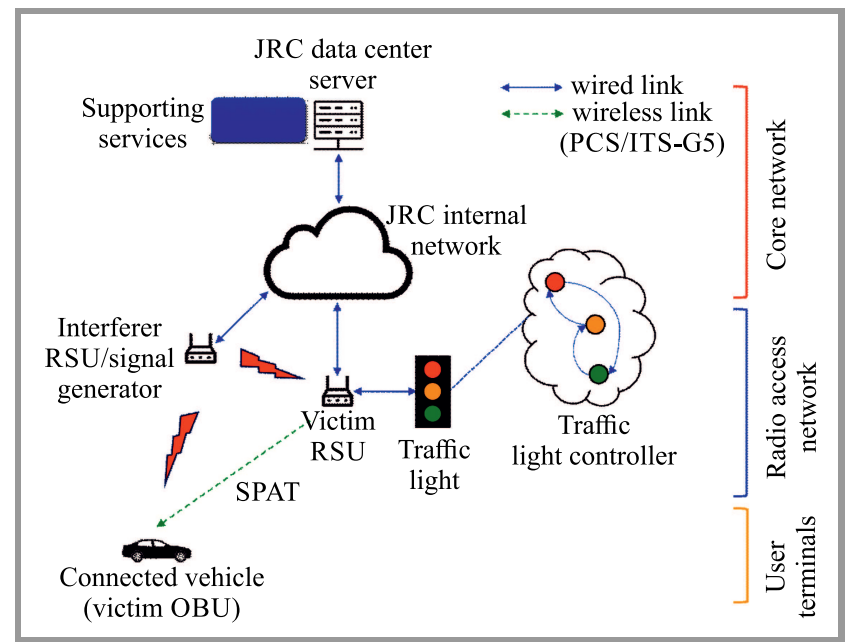

Fig. 3. Architecture of the GLOSA performance test. a finite state machine (FSM) (if virtual). The RSU will periodically broadcast signal phase and timing messages (SPAT) (e.g. every $100 \mathrm{~ms}$ ) to all vehicles nearby;

- An ITS-G5 RSU installed at the traffic light location (if physical) or running on / communicating with the FSM (if virtual). The RSU will periodically broadcast signal phase and timing messages (SPAT) (e.g. every $100 \mathrm{~ms}$ ) to all vehicles nearby;

- Two On-Board Units (OBUs) (one ITS-G5, one LTE$\mathrm{V} 2 \mathrm{X}$ ) deployed in the test vehicles. The OBUs will receive and process the SPAT messages locally to compute the relevant GLOSA information in various forms, e.g. time remaining until transition to the next traffic light phase, optimum speed to reach the traffic light in the green phase, etc. Once computed, the GLOSA information will be relayed to an on-board laptop (or UI device), where it will be communicated, both visually and audibly, to the driver;

- The JRC internal communications network will provide connectivity between the RSUs and various supporting services running in the JRC data center, e.g. the experiment management console, the traffic light controller, the log server, etc.;

- Physical/virtual servers in the JRC data center running the above-mentioned supporting services. For the purpose of implementing and experimentally evaluating this use case, these servers may be provisioned either physically or with the use of virtual machines (VMs).

During the course of the performance tests, both ITS-G5 and LTE-V2X units are exposed to external harmful interference. This interference will be generated either by ITS-G5 or LTE-V2X units themselves (depending on the specific test being run) or, alternatively, by an external signal generator.

It needs to be mentioned that the RSU also broadcasts MAP messages. The vehicles present nearby may receive these messages on their OBUs and process them locally along, inter alia, with their own positioning, speed and direction data. By doing so, on-board V2X modules can notify drivers of the optimum speed allowing to reach the upcoming traffic light during the green phase or, alternatively, can notify them that the traffic light will nevertheless transition to red imminently. The dynamic information is disseminated using the SPAT V2X I2V message and contains the time remaining until the traffic light changes and information about the recommended speed, applicable to a group of entry lanes. MAP and SPAT messages are already standardized [36] and profiled [37]. However, the interpretation of their content at the receiving side (cooperative vehicles), and the relation between this content and the actual current status of the traffic light controller may still lead to confusion. 
Knowledge on how to interpret the SPAT content at the receiving side is particularly critical in the case of connected automated vehicles (CAVs) [38]. A CAV is assumed to be an ETSI ITS G5 [11] equipped vehicle with automated driving capabilities. In this sense, it is an improved version of a cooperative vehicle. It uses vehicle sensors and $\mathrm{V} 2 \mathrm{X}$ transmissions to monitor its surroundings, as well as V2X communications to interact with other cooperative vehicles, CAVs and with cooperative intersections (CIs) [39]. Such a vehicle relies on the flow of information obtained for trajectory and maneuver planning, platoon organization and for implementation of improved, advanced driver assistance system (ADAS) functionalities. In a broader sense, a CAV may be a vehicle of any type. Multiple CAVs can form a vehicle platoon. In fact, the automated behavior of CAVs approaching a cooperative intersection (CI) will depend strongly on the information provided via the SPAT message. It is assumed that a CI has the form of an ETSI ITS G5 [11] equipped traffic light controller. In addition to such infrastructure sensors as inductive loops and cameras relied upon to detect all types of road users, the CI uses V2I communication to exchange data with cooperative vehicles and CAVs. The CI uses the flow of information for adaptive optimization of traffic light timing, simultaneously taking into account additional policy parameters, such as specific traffic priorities and demands pertaining to vulnerable road users [40]. In addition, it uses V2I communications to interact with cooperative vehicles, for example to provide recommendations concerning speed values and lanes to be chosen. Multiple CIs may be connected to enable traffic light coordination along a specific traffic corridor.

Based on the correct interpretation of the SPAT content, and together with other environmental information obtained via the onboard sensors, CAVs will decide whether to adjust the speed to the suggested value or to prepare for stopping. As far as the 5G-DRIVE context is concerned, GLOSA is an attractive V2X use case allowing to improve traffic flow in urban areas. It provides drivers with optimum speed recommendation as they approach an intersection with traffic lights. The recommendation, usually displayed on the car's

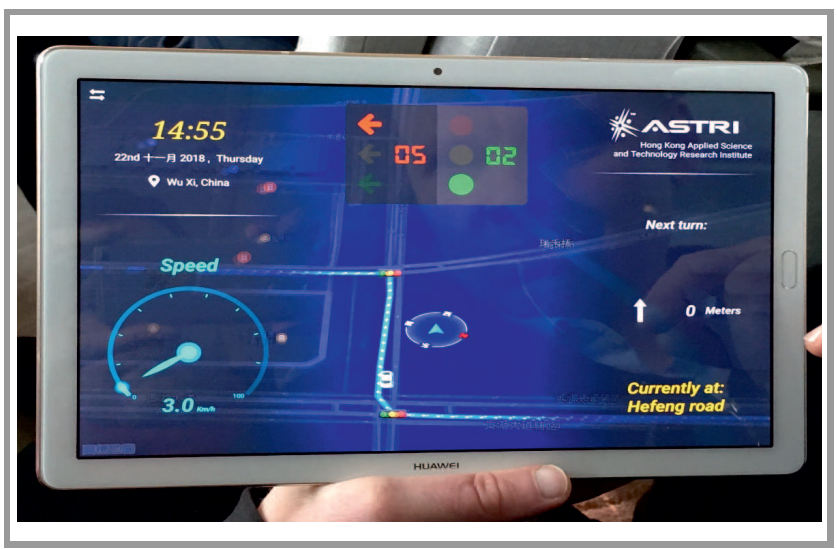

Fig. 4. LTE-V2X GLOSA demonstration by China Mobile, Huawei and ASTRI in the context of the 5G-DRIVE project. dashboard, will instruct the driver to maintain the current speed, slow down or speed up. GLOSA may also provide time-to-green information when the vehicle is stationary at a traffic light. GLOSA takes advantage of real-time traffic sensing and infrastructure information that may then be relayed to vehicles aiming to reduce fuel consumption. The GLOSA system may operate in the manner presented in Fig. 4.

\subsection{KPIs and Performance Test Procedures}

Since 5G-DRIVE aims to compare the benefits of $5 \mathrm{G}$ in Europe and China, some common test scenarios have been planned for implementation both in China and at the European trial sites. Common KPIs are defined to measure the same parameters and to compare the results. The trials aim to capture, analyze and discuss the following KPIs that can be either Quality of Service (QoS) or Quality of Experience (QoE) metrics. The former group includes: transmission range, average data rate, total message/s per channel and latency, i.e. the contribution of the radio access network to the total elapsed time, measured from the instant the RSU sends a packet to the moment when the OBU receives it. The packet error rate (PER) is measured as well, i.e. the ratio of packets unsuccessfully received by the OBU vs. the total number of packets sent by the RSU (percentagewise). Other parameters include spectral efficiency and channel busy ratio (CBR, defined as the ratio between the time the channel is sensed as busy and the total observation time). It is a measure of channel load perceived by a vehicle, and depends on the number of vehicles within its transmission range and on their individual message generation rates, which makes it a metric suitable for increasing packet delivery performance.

Other related KPIs belonging to the same category include the following: co-channel and adjacent-channel interference and duty cycle. QoE metrics, in turn, include the following: deployment complexity, as well as ease of configuration and setup. Chinese KPIs are more exhaustive, since a greater emphasis is attached to network optimization, whereas 5G-DRIVE is application-driven. Because of the low volume of data/information transmitted in GLOSA (traffic light state machine timing data), KPIs such as peak/user-perceived data rate are not a critical concern in the context of this specific use case. Instead, the following most relevant service-level performance indicators have been defined for GLOSA [41]: PER and latency, i.e. the contribution of the radio access network to the total elapsed time, measured from the instant the RSU sends a packet to the moment when the OBU receives it.

For the purpose of the GLOSA trial, a commercial ITSG5 roadside unit has been deployed at the JRC Ispra trial site/campus, covering a section of its internal road. The RSU sits at the junction of two suburban-type roads that are 420 and $220 \mathrm{~m}$ long, respectively, at a height of approximately $10 \mathrm{~m}$. In addition, the RSU is connected to the trialsite's internal network infrastructure to allow remote configuration, management and traffic monitoring. The com- 
mercial RSU runs a Linux-based operating system, thus allowing the execution of custom user space applications (such as a virtual traffic light for the GLOSA service). This setup is presented in Fig. 5.

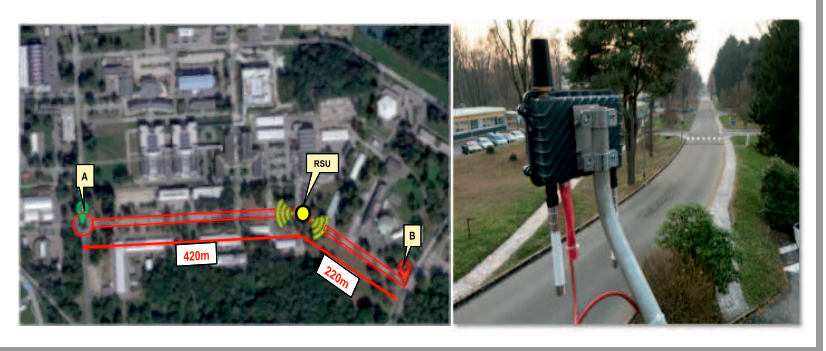

Fig. 5. ITS G5 RSU deployed in the Ispra campus.

The GLOSA setup described above is to be extended by deploying an LTE-V2X unit co-located with the ITS-G5 RSU. The LTE-V2X unit will also be connected to the campus' internal network for remote configuration, management and traffic monitoring purposes. In the context of the GLOSA trial, both technologies will be tested in a mutually-exclusive fashion to avoid harmful interference between them. The C-ITS road infrastructure described above is complemented by two OBUs (one ITS-G5, one LTE-V2X) co-located in a test vehicle for the purpose of the GLOSA trials. The OBUs are connected to a laptop for experiment configuration and traffic monitoring purposes.

As far as the specific test setup is concerned, coexistence tests will feature two C-ITS systems: an interferer and a victim. Each system will comprise two C-ITS units (ITSG5 or LTE-V2X, depending on the particular test scenario). The victim system units will actively exchange C-ITS messages (e.g. standard CAM broadcasts) used in regular CITS operation. By contrast, the interferer system units will initially be set to operate in the idle mode (i.e. without broadcasting any C-ITS messages via the $5.9 \mathrm{GHz}$ channel). Later, they will start sending C-ITS application messages of a user-controlled length (len $\mathrm{msg}_{\mathrm{g}}$, in bytes) and packet rate $\left(\mathrm{R}_{\mathrm{pkt}}\right.$, in packets/s) with the aim of causing harmful interference affecting the victim system. The following KPIs will be continuously monitored and recorded in the victim system.

The proposed GLOSA performance test procedure required that the following steps be taken:

- the vehicle starts driving towards the RSU at 30$40 \mathrm{~km} / \mathrm{h}$;

- the vehicle enters RSU's transmission range and the OBU starts receiving SPAT messages;

- the GLOSA client app successfully processes traffic light phase information encapsulated in SPAT messages. The in-vehicle UI displays the traffic light change-of-phase timer;

- traffic light phase and SPAT phase information are synchronized. The success criterion implicates that the traffic light phase changes when the in-vehicle timer expires;

- previous steps are repeated by setting $\operatorname{len}_{\mathrm{msg}}=$ $(50,100,200,300,400,500)$ bytes, and by setting $\mathrm{R}_{\mathrm{pkt}}=(300,600,650,700,750,800)$ packets/s, in the interferer RSU or in an external signal generator. Now the success criterion implicates that for higher values of len $\mathrm{msg}_{\mathrm{m}}$ and $\mathrm{R}_{\mathrm{pkt}}$, the interferer system blocks communication between victim units.

As the procedure described above is a performance test, no actual pass/fail success criteria are set. The goal of the test is to evaluate resilience of ITS-G5 and LTE-V2X units against external harmful interference. The previously notified KPIs will be evaluated.

\section{Discussion}

It is quite interesting to mention that the actual GLOSA use case has also been examined in parallel with the C$\mathrm{V} 2 \mathrm{X}$ intelligent intersection use case, although the latter has more strict requirements. The purpose of such an approach was to ensure joint applicability and to extend visibility and market impact by using the same infrastructure and/or equipment. Offering a greater variety of services to the users and/or other market actors involved was another of the objectives pursued.

The tests will comprise two main activities. Firstly, there will be a subset of compliance tests specified in the ETSI Harmonized Standard for radio-communications equipment operating in the 5855-5925 MHz frequency band (ETSI EN 302 571) using standalone ITS-G5 and LTE-V2X devices [42]. The purpose of these tests is to verify the conformance of commercial/pre-commercial V2X devices with the European standard for radio transmissions in the $5.9 \mathrm{GHz}$ band - a market-entry condition as laid down in the EU Radio Equipment Directive. Additionally, they will also help acquire the hands-on skills that are necessary to operate $\mathrm{V} 2 \mathrm{X}$ equipment in a test environment. In the second phase, the aim will be to evaluate co-channel and adjacent-channel interference of ITS-G5 over LTE-V2X devices and vice-versa, both in conducted mode (laboratory) and in the anechoic chambers of the JRC Ispra site.

The subset of the conformance tests defined in ETSI EN 302571 allows to verify the following:

Frequency stability: The goal of this test is to ensure that V2X equipment can operate on the specific carrier center frequencies $\left(f_{c}\right)$ that correspond to the nominal carrier frequencies covering the $5860-5920 \mathrm{MHz}$ band in $10 \mathrm{MHz}$ steps. The actual carrier center frequency for any of the above $10 \mathrm{MHz}$ channels shall be maintained within the range $f_{c} \pm 20 \mathrm{ppm}$.

RF output power: The purpose of this test is to ensure that the mean equivalent isotropically radiated power (EIRP) of V2X equipment during transmission bursts does not exceed $33 \mathrm{dBm}$. 
Power spectral density: The aim of this test is to ensure that the power spectral density (PSD) of the V2X device during transmission bursts does not exceed $23 \mathrm{dBm} / \mathrm{MHz}$ EIRP.

Transmit power control (TPC): TPC is a scheme supporting coexistence with the European Committee for Standardization (CEN) dedicated short-range communication (DSRC) at toll plazas and is used as a single mechanism by decentralized congestion control (DCC) solutions to reduce communication channel congestion. The goal of this test is to ensure that TPC operates as expected in the presence of CEN DSRC devices, as well as during DCC.

Transmitter unwanted emissions: The purpose of this test is to ensure that $\mathrm{V} 2 \mathrm{X}$ devices respect the radio frequency (RF) power limits for radio frequency emissions outside the $5 \mathrm{GHz}$ ITS frequency band (outside of 5855-5925 MHz).

Decentralized Congestion Control (DCC): DCC is a mandatory ITS-G5 mechanism to ensure that the radio channel is not congested by too many transmissions within a certain geographical range. The mechanism is such that the equipment adapts its transmission behavior dynamically, based on how occupied the channel is at a given moment. The aim of this test is to ensure that DCC operates as expected in the presence of interferer signals in the $5.9 \mathrm{GHz}$ band.

As mentioned above, several ITS-G5/LTE-V2X coexistence tests will be performed as well to evaluate the degree of harmful interference between commercial/pre-commercial off-the-shelf ITS-G5 and LTE-V2X equipment in the absence of any active coexistence mechanisms in their radio protocol stacks. The outcome of these experiments will provide a baseline scenario for further tests once specific coexistence mechanisms have been specified, implemented and deployed in the ITS-G5 and LTE-V2X radio protocol stacks.

The main conceptual differences consist in the fact that in China the trials are LTE network-based, whereas the European ones have been performed with the use of PC5 devices, while also relying on an LTE network. The other distinction is that European V2X applications use the decentralized environmental notification messages (DEMN) message format, whereas in China, BSM type messages are used. The KPIs have been selected to offer complementary measurements between the $5 \mathrm{G}$ large-scale trial in China and the 5G-DRIVE project in Europe. However, some application-specific measures related to ETSI- and SAE-standardized message deliveries between the digital infrastructure and the vehicle need to be taken into consideration as well. The aims of the Chinese and the European V2X tests are slightly different. Chinese trials are led by a telecom operator and, therefore, they focus more on network side operation and on latencies in different communication planes. As expected, in Europe the tests focus on pure communication and cover also communication delays between cloud services and vehicle components (OBUs, message parser, etc.), since the main objective is to understand the benefits of deploying intelligent vehicle compo- nents. The packet error rate target in effect in Europe is less than $5 \%$, whereas the Chinese target is less than $10 \%$. Latency targets are equivalent, as they equal $100 \mathrm{~ms}$. In China, latency is divided between control and data planes (CP and DP) only, whereas European V2X trials do distinguish different latency sources.

\section{Acknowledgement}

This paper has been drawn up based on the research performed under the H2020 5G-DRIVE (Harmonized Research and Trials for Service Evolution between EU and China) project, funded by the European Commission under Grant Agreement no. 814956.

\section{References}

[1] 5G-PPP, "5G strategic deployment agenda for connected and automated mobility in Europe" [Online]. Available: https://5g-ppp.eu/ wp-content/uploads/2020/10/20201002_5G_SDA_for_CAM_Final.pdf

[2] "Report ITU-R M.2243 (00/2011): Assessment of the global mobile broadband deployments and forecasts for International Mobile Telecommunications", Int. Telecommunication Union [Online]. Available: https://www.itu.int/dms_pub/itu-r/opb/rep/ R-REP-M.2243-2011-PDF-E.pdf

[3] J. G. Andrews et al., "What will 5G be?", IEEE JSAC, Special issue on $5 G$ Wireless Commun. Systems, vol. 32, no. 6, pp. 1065-1082, 2014 [Online]. Available: https://arxiv.org/pdf/1405.2957

[4] L. Figueiredo et al., "Towards the development of intelligent transportation systems", in Proc. IEEE Intelligent Transportat. Systems, Oakland, CA, USA, 2001, pp. 1206-1211 (DOI: 10.1109/ITSC.2001.948835).

[5] ETSI, "TS 103723 V1.2.1 (2020-11): intelligent transport systems (ITS); profile for LTE-V2X Direct Communication”, 2020 [Online]. Available: http://www.etsi.org/deliver/etsi_ts/103700_103799/ 103723/01.02.01_60/ts_103723v010201p.pdf

[6] A. Festag, "Cooperative intelligent transport systems in Europe", IEEE Vehicular Technol. Mag., vol. 12, no. 2, pp. 89-97, 2017 (DOI: 10.1109/MVT.2017.2670018).

[7] ISO/CEN, "Cooperative intelligent transport systems (C-ITS). Guidelines on the usage of standards", 2020 [Online]. Available: https://www.itsstandards.eu/app/uploads/sites/14/2020/10/ C-ITS-Brochure-2020-FINAL.pdf

[8] NGMN, "V2X White Paper", 2018 [Online]. Available: https://www.ngmn.org/wp-content/uploads/ V2X_white_paper_v1_0-1.pdf

[9] GSMA, "Connecting vehicles - today and in the $5 \mathrm{G}$ era with C-V2X" [Online]. Available: https://www.gsma.com/iot/wp-content/ uploads/2019/08/Connecting-Vehicles-Today-and-in-the-5G-Erawith-C-V2X.pdf

[10] H. Bhatia, "125 Million+ connected cars shipments by 2022; 5G cars by 2020", Counterpoint, 2018 [Online]. Available: https://www.counterpointresearch.com/ 125-million-connected-cars-shipments-2022-5g-cars-2020/

[11] ETSI, "EN 302663 V1.3.1 (2019-10): intelligent transport systems (ITS); ITS-G5 access layer specification for intelligent transport systems operating in the $5 \mathrm{GHz}$ frequency band" [Online]. Available: https://www.etsi.org/deliver/etsi_en/302600_302699/ 302663/01.03.01_30/en_302663v010301v.pdf

[12] 3GPP, “TS 22.185 v16.0.0 (2020-07): service requirements for V2X services" [Online]. Available: https://www.3gpp.org/ftp//Specs/ archive/22_series/22.185/

[13] 5G-DRIVE H2020 5G-PPP Project [Online]. Available: https://5g-drive.eu 
[14] I. P. Chochliouros et al., "Testbeds for the implementation of $5 \mathrm{G}$ in the European Union: the innovative case of the 5G-DRIVE project", in Proc. Artificial Intelligence Applications and Innovations, 2019, pp. 78-92, (DOI: 10.1007/978-3-030-19909-8_7).

[15] A. Kostopoulos et al., "5G trial cooperation between EU and China", in Proc. IEEE Int. Conf. on Commun. Workshops (ICC 2019 Workshops), 2019, Shanghai, China, pp. 1-6 (DOI: $10.1109 / \mathrm{ICCW} .2019 .8756985)$.

[16] ETSI, "TS 138300 V15.3.1 (2018-10): 5G; NR; overall description" [Online]. Available: https://www.etsi.org/deliver/etsi_ts/ 138300_138399/138300/15.03.01_60/ts_138300v150301p.pdf

[17] R. Verdone and A. Manzalini, "5G experimental facilities in Europe", NetWorld 2020 ETP [Online]. Available:

https://www.networld2020.eu/wp-content/uploads/2016/03/ 5G-experimentation-Whitepaper-v11.pdf

[18] J. Wang, Y. Shao, Y. Ge, and R. Yu, "A survey of vehicle to everything (V2X) testing", Sensors, vol. 19, no. 2, 2019 (DOI: $10.3390 / \mathrm{s} 19020334)$.

[19] IEEE, "IEEE 802.11p-2010: IEEE Standard for Information Technology (IT) - Local and metropolitan area networks - Specific requirements - Part 11: Wireless LAN Medium Access Control (MAC) and Physical Layer (PHY) Specifications Amendment 6: Wireless Access in Vehicular Environments" [Online]. Available: https://standards.ieee.org/standard/802_11p-2010.html

[20] 5GAA, "An assessment of LTE-V2X (PC5) and 802.11p direct communications technologies for improved road safety in the EU", 2017 [Online]. Available: http://5gaa.org/wp-content/uploads/2017/ 12/5GAA-road-safety-FINAL2017-12.05.pdf

[21] ETSI, "EN 302665 V1.1.1 (2010-09): intelligent transport systems (ITS); communication architecture" [Online]. Available: https://www.etsi.org/deliver/etsi_en/302600_302699/302665/ 01.01.01_60/en_302665v010101p.pdf

[22] 3GPP, "TR 21.914 v14.0.0: release 15 description; summary of Rel14 work items", 2018 [Online]. Available: https://www.3gpp.org/ $\mathrm{ftp} /$ Specs/archive/21_series/21.914/

[23] 3GPP, "TR 21.915 v0.4.0: release 15 description; summary of Rel15 work items," 2018 [Online]. Available: https://www.3gpp.org/ftp/Specs/archive/21_series/21.915/

[24] 3GPP, "TR 21.916 v1.0.0: release 16 description; summary of Rel16 work items," 2020 [Online]. Available: https://www.3gpp.org/ftp/Specs/archive/21_series/21.916/

[25] S. Kekki et al., "MEC in 5G Networks, ETSI White paper", European Telecommunications Standards Institute (ETSI), no. 28, 2018 [Online]. Available: https://www.etsi.org/images/files/ ETSIWhitePapers/etsi_wp28_mec_in_5G_FINAL.pdf

[26] ETSI, "ETSI GS MEC 003 V1.1.1: mobile edge computing (MEC); framework and reference architecture" [Online]. Available: https://www.etsi.org/deliver/etsi_gs/MEC/001_099/003/01.01.01_60/ gs_MEC003v010101p.pdf

[27] J. Santa, F. Pereniguez-Garcia, A. Moragón, and A. Skarmeta, "Experimental evaluation of CAM and DENM messaging services in vehicular communications", Transportat. Res. Part C: Emerging Technol., vol. 46, pp. 98-120, 2014 (DOI: 10.1016/j.trc.2014.05.006).

[28] S. I. Guler, M. Menendez, and L. Meier, "Using connected vehicle technology to improve the efficiency of intersections", Transportat. Res. Part C: Emerging Technol., vol. 46, pp. 121-131, 2014 (DOI: 10.1016/j.trc.2014.05.008).

[29] A. Stevanovic, J. Stevanovic, and C. Kergaye, "Green light optimized speed advisory systems: impact of signal phasing information accuracy", J. of the Transportat. Res. Board, vol. 2390, no. 1, pp. 53-59, 2013 (DOI: 10.3141/2390-06).

[30] K. Katsaros, R. Kernchen, M. Dianati, and D. Rieck, "Performance study of a green light optimized speed advisory (GLOSA) application using an integrated cooperative ITS simulation platform", in Proc. Int. Wireless Commun. and Mobile Comput. Conf. (IWCMC), Istanbul, Turkey, 2011, pp. 918-923 (DOI: 10.1109/IWCMC.2011.5982524).
[31] T. Tielert et al., "The impact of traffic-light-to-vehicle communication on fuel consumption and emissions", in Proc. Internet of Things 2010 Conf. (IoT2010), Tokyo, Japan, 2010, pp. 1-8 (DOI: 10.1109/IOT.2010.5678454).

[32] M. Chao-Qun, H. Hai-Jun, and T. Tie-Qia, "Improving urban traffic by velocity guidance", in Proc. Int. Conf. on Intelligent Comput. and Automation (ICICTA-2008), vol. 2, pp. 383-387 (DOI: 10.1109/ICICTA.2008.288).

[33] C-ROADS [Online]. Available: https://www.c-roads.eu/platform.html

[34] M. Lu et al., "C-ITS (cooperative intelligent transport systems) deployment in Europe - challenges and key findings", in Proc. ITS World Congress, pp. 1-10, 2018.

[35] B. Asadi and A. Vahidi, "Predictive cruise control: Utilizing upcoming traffic signal information for improving fuel economy and reducing trip time", IEEE Transac. on Control Systems Technol., vol. 19 , no. 3, pp. 707-714, 2010 (DOI: 10.1109/TCST.2010.2047860).

[36] SAE, "SAE J2735_201603, dedicated short range communications (DSRC) message set dictionary", 2016 [Online]. Available: https://www.sae.org/standards/content/j2735_201603/

[37] Talking Traffic consortium, Dutch profiles and ITF, "Smart mobility community for standards and practices", 2019 [Online]. Available: http://www.smartmobilitycommunity.eu/ talking-traffic-dutch-profiles-and-itf.

[38] M. Galvani, "History and future of driver assistance", IEEE Instrumentation Measurement Mag., vol. 22, no. 1, pp. 11-16, 2019 (DOI: 10.1109/MIM.2019.8633345).

[39] L. Chen and C. Englund, "Cooperative intersection management: A survey", IEEE Transac. on Intelligent Transport. Systems, vol. 17, no. 2, pp. 570-586, 2015 (DOI: 10.1109/TITS.2015.2471812).

[40] M. Hafner, D. Cunningham, L. Caminiti, and D. Del Vecchio, "Cooperative collision avoidance at intersections: Algorithms and experiments", Trans. Intel. Transportation Systems, vol. 14, no. 3, pp. 1162-1175, 2013 (DOI: 10.1109/TITS.2013.2252901).

[41] 5G-DRIVE Project, "Deliverable D4.2: Joint specifications for V2X trials", 2018.

[42] ETSI, "EN 302 571v2.1.1 (2017-02): Intelligent Transport Systems (ITS); Radiocommunications equipment operating in the $5855 \mathrm{MHz}$ to $5925 \mathrm{MHz}$ frequency band; Harmonised Standard covering the essential requirements of article 3.2 of Directive 2014/53/EU" [Online]. Available: https://www.etsi.org/deliver/etsi_en/302500_ 302599/302571/02.01.01_60/en_302571v020101p.pdf

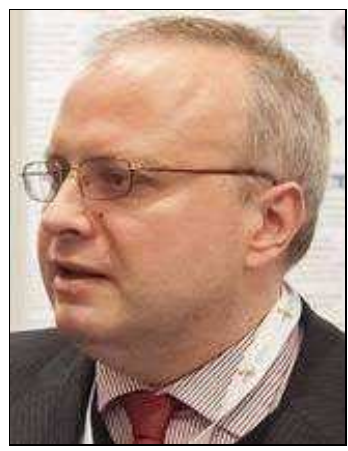

Ioannis P. Chochliouros graduated from the Dept. of Electrical Engineering at the Polytechnic School of the Aristotle University of Thessaloniki, Greece, holding also an M.Sc. (D.E.A.) and a Ph.D. from Université Pierre et Marie Curie (Paris VI), France. His practical experience as an engineer has been mainly in telecommunications, as well as in various construction projects in Greece and in the wider Balkan area. Since 1997, he has been working at the Competition Department and as an engineer-consultant of the Chief Technical Officer of the Hellenic Telecommunications Organization (OTE). He was closely involved in OTE's major national and international business activities, as 
a specialist-consultant for technical and regulatory affairs, especially for the evaluation and the adoption of innovative e-infrastructures and e-services in Greece and abroad. He has also served as the Head of Technical Regulations Department of OTE's Division for Standardization and Technical Regulations, representing OTE in international standardization bodies, and has been involved in a great variety of projects regarding European and international standardization, with an emphasis placed on modern technologies. In addition, he has also worked as an independent consultant, participating in several European and/or international research and business studies. Since 2005, he has been the Head of OTE's Fixed Network R\&D Programs Section and has been involved in different national, European and international R\&D projects and market-oriented activities, many of which have received international awards. During his professional career, he has participated - either as a coordinator or a scientist-researcher - in more than 62 European and national research programs (IST, STREP, EURESCOM, FP6, FP7, ESA, H2020 and the 5G-PPP phases I-III). He is the author/co-author of three international books and has published more than 250 distinct scientific or business papers/reports in international papers.

(iD) https://orcid.org/0000-0002-4208-1676

E-mail: ichochliouros@oteresearch.gr

Hellenic Telecommunications Organization

Athens, Greece

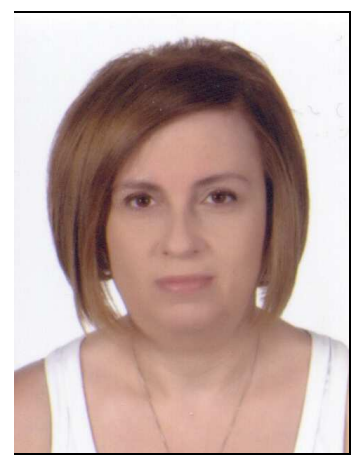

Anastasia S. Spiliopoulou is a lawyer and a Member of the Athens Bar Association. She also holds a post-graduate diploma from the Law School of the National and Kapodistrian University of Athens, Greece. She has many years of professional experience in telecommunications and IT-related issues and has been involved in many legal cases related to regulatory issues, as well as to the deployment and operation of modern electronic communication networks, and to the provision of communications services. She is OTE's (Hellenic Telecommunications Organization) expert for a great variety of regulatory issues affecting both European and national policies. Apart from her expertise in regulatory matters concerned with networking and service offering, she specializes also in privacy- and data protection-related issues. She is the author/coauthor of more than 110 papers published in international papers and has participated in numerous conferences, acting in the capacity of the invited speaker on several occasions.

E-mail: aspiliopoul@ote.gr

Hellenic Telecommunications Organization Athens, Greece

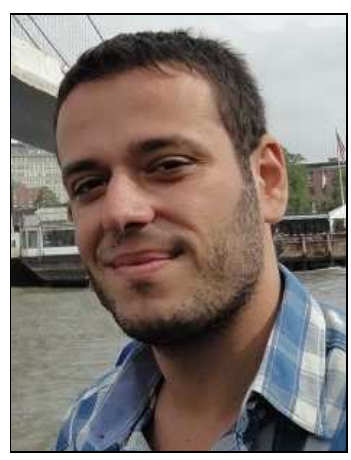

Alexandros Kostopoulos holds two M.Sc. degrees in Telecommunications (University of Athens) and in Computer Science (University of Piraeus). He received his Ph.D. from Athens University of Economics and Business (AUEB) in 2013. He was a visiting lecturer at AUEB, as well as a postdoctoral researcher at the Institute of Computer Science, in Forth. He is currently working at the Research and Development Department of OTE (Hellenic Telecommunications Organization), focusing on European and national research projects. He has been involved, inter alia, in various 5G-PPP projects (phases I-III). He has published several papers in scientific journals and conferences and has joined numerous events in the capacity of a speaker.

E-mail: alexkosto@oteresearch.gr

Hellenic Telecommunications Organization

Athens, Greece

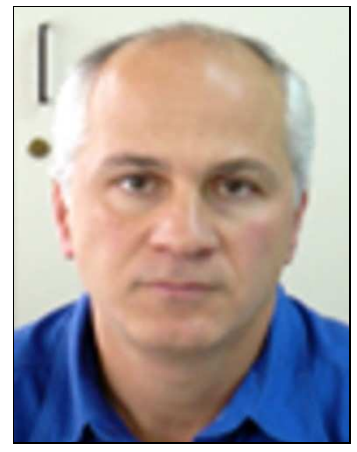

George Agapiou received his Diploma in Electrical Engineering from the University of Louisville, Kentucky, in 1985, as well as M.Sc. and Ph.D. degrees in Electrical Engineering from the Georgia Institute of Technology, in 1987 and 1991, respectively. From 1984 to 1985 , he worked at Philip Morris at Louisville, Kentucky as a Maintenance Engineer. Since 1996 he has been a telecom engineer at OTE and COSMOTE, where he worked on various European projects in the area of Mobile and Optical Communications. Currently, he holds the position of the Head of Core Lab Testing. He has participated in various IST, STREP, EURESCOM, FP6, FP7, ESA, H2020 and 5G-PPP (phases I-III) projects and has published more than 100 papers in scientific journals and proceedings and has been a co-author of three technical books.

E-mail: gagapiou@ oteresearch.gr

Hellenic Telecommunications Organization

Athens, Greece

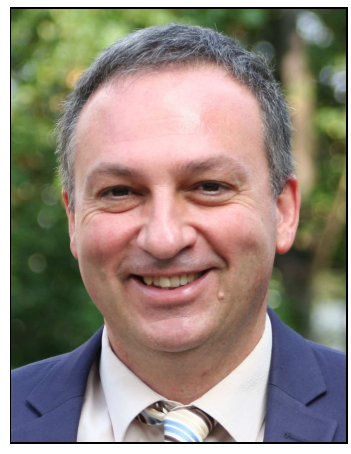

Pavlos Lazaridis is a Professor of Electronic and Electrical Engineering at the University of Huddersfield, UK. He received his Electrical Engineering degree from the Aristotle University of Thessaloniki, Greece, in 1990, the M.Sc. degree in Electronics from Université Pierre et Marie Curie, Paris 6, France, in 1992, and the Ph.D. 
degree in Electronics and Telecommunications from Ecole Nationale Supérieure des Télécommunications (ENST) and Paris 6, Paris, in 1996. From 1991 to 1996, he researched semiconductor lasers, wave propagation, and nonlinear phenomena in optical fibers for the Centre National d'Etudes des Télécommunications (CNET) and was teaching at ENST. In 1997, he became the Head of the Antennas and Propagation Laboratory, TDF-C2R Metz (Télédiffusion de France/France Télécom Research Center), where he conducted research on antennas and radio-coverage for cellular mobile systems (GSM), digital audio broadcasting (DAB), and digital video broadcastingterrestrial (DVB-T). From 1998 to 2002, he was with the European Patent Office, Rijswijk, the Netherlands, as a Senior Examiner in the field of Electronics and Telecommunications. He is leading the EU Horizon 2020 projects titled ITNMOTOR5G and RISE-RECOMBINE, for the University of Huddersfield, UK.

E-mail: P.Lazaridis@hud.ac.uk

Department of Engineering and Technology

University of Huddersfield

Huddersfield, United Kingdom

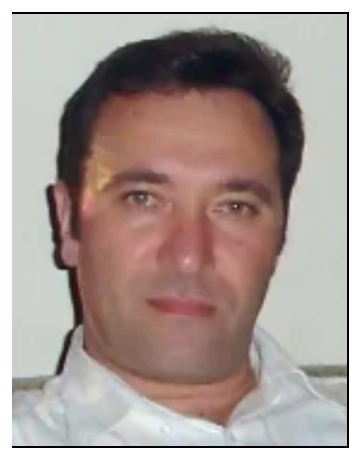

Zaharias D. Zaharis received his B.Sc. in physics, M.Sc. in electronics, Ph.D. and a Diploma degree in Electrical and Computer Engineering from the Aristotle University of Thessaloniki, Thessaloniki, Greece, in 1987, 1994, 2000, and 2011, respectively. From 2002 to 2013 , he was with the administration of the telecommunications network, Aristotle University of Thessaloniki, and since 2013 he has been with the Department of Electrical and Computer Engineering of the same university. His current research interests include design and optimization of antennas and microwave circuits, signal processing on smart antennas, development of evolutionary optimization algorithms, and neural networks.

E-mail: zaharis@auth.gr

Aristotle University of Thessaloniki

Thessaloniki, Greece

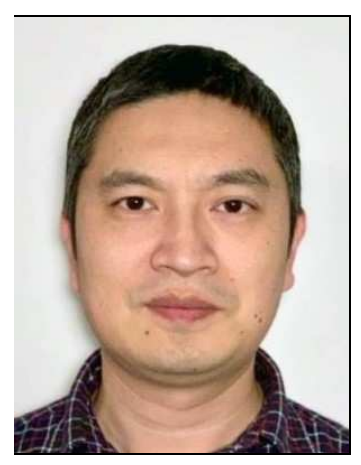

Tao Chen received his Ph.D. degree in telecommunications engineering from the University of Trento, Italy in 2007. Then, he joined the VTT Technical Research Centre of Finland and currently he is a senior researcher focusing on the area of connectivity. He is a docent (adjunct professor) at the University of Jyväskylä, and a pro- ject coordinator for 5G PPP COHERENT. His current research interests include software-defined networking for $5 \mathrm{G}$ mobile networks, massive IoT in 5G, dynamic spectrum access, energy efficiency and resource management in heterogeneous wireless networks, and social-aware mobile networks.

E-mail: Tao.Chen@vtt.fi

VTT Technical Research Centre of Finland

Espoo, Finland

Athanassios Dardamanis is the CEO of Smartnet S.A. and has graduated from the IT Dept. of the National and Kapodistrian University of Athens, Greece. He has extensive experience in a variety of technical implementation and design activities and has worked with operators, enterprises, organizations and public bodies in Greece. He has successfully implemented numerous technical projects for various customers and has supported the company's strive towards widening the scope of its presence in the area of modern technological applications. He has extensive administrative and technical experience obtained while working in the capacity of a top executive of several very well-known IT companies in the Hellenic market (such as Altec and Alcatel).

E-mail: adardamanis@smartnet.gr

SmartNet

Athens, Greece

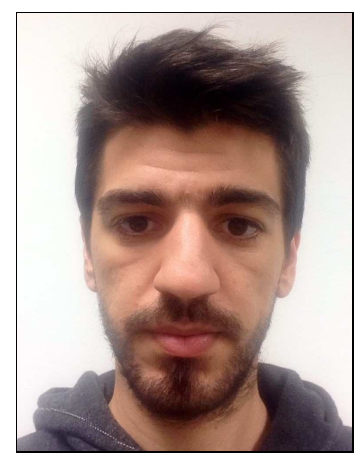

Michail-Alexandros Kourtis received his Ph.D. from UPV/ EHU in 2018 and his Diploma and M.Sc. in Computer Science from the Athens University of Economics and Business, in 2011 and 2013, respectively. Since 2015, he has worked on cybersecurity applications for Network Function Virtualization, as well as on the definition of privacy and security risk metrics for virtualized infrastructures. His research interests include QoE, QoS, video processing, video quality assessment, image processing, LTE, 5G, network function virtualization, and software-defined networks. He is a contributor to the OPNFV open-source project known as Yardstick, and an active member, participant and contributor at the IETF NFVRG. He is also a TPC member and a reviewer at various conferences and journals. He has joined several EU-funded projects, with some of them implemented within the framework of 5G-PPP (phases I-III).

E-mail: akourtis@orioninnovations.gr ORION Innovations Private Company

Athens, Greece 


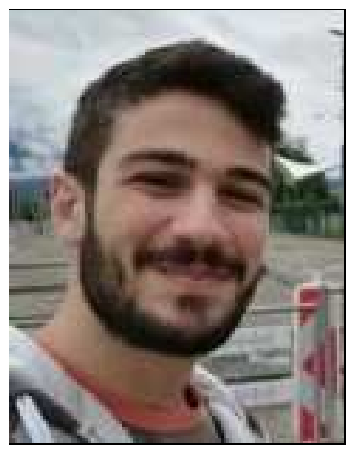

Marinos Agapiou has completed his B.S. at the National Kapodistrian University of Athens and is currently complementing his MBA at the Athens University of Economics and Business in Greece. He is the author of several papers dealing with telecommunications and market analysis.

E-mail: minosagap@gmail.com

Independent Consultant

Athens, Greece

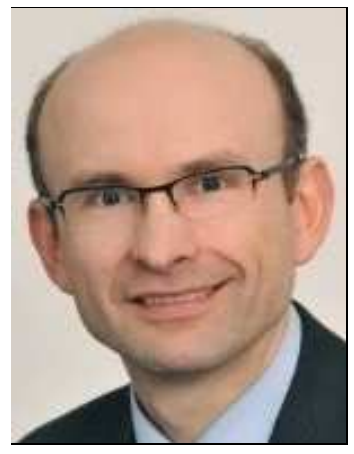

Uwe Herzog is a Program Manager at Eurescom. He has more than 20 years of professional experience. He has been involved in a number of EC research projects as project coordinator, work-package leader and in other roles, including projects such as WINNER I and II, XIFI, CONCORD, ENVIROFI, eMobility NetWorld, UbiSec\&Sens, WSAN4CIP, EURO-5G, SPEED-5G, ORPHEUS, Euro-5G and To-Euro-5G. Uwe has also been an evaluator and reporter in the FP7 and H2020 Call evaluation process in the area of Future Networks and SME-Instrument on Open Disruptive Innovation. He holds an M.Sc. degree in electrical engineering from the
University of Chemnitz, Germany, and an MBA from the University of Mannheim and ESSEC Business School Paris.

E-mail: herzog@eurescom.eu

Eurescom

Heidelberg, Germany

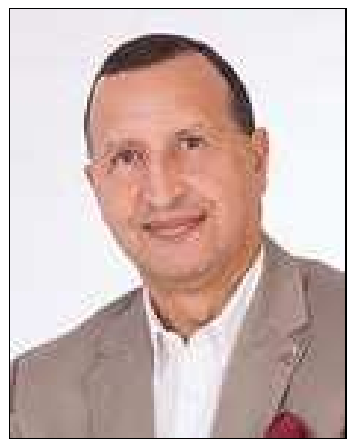

Latif Ladid is the Founder and current President of the IPv6 Forum and has been a Board Member of 3GPP since 1999. He is also the Chairman of the European IPv6 Task Force, the Chairman of the $5 \mathrm{G}$ World Alliance, and the Chairman of the following technical Committees: IEEE COMSOC IoT subCommittee, IEEE COMSOC 5G subCommit, and ETSI IPv6 ISG (including $5 \mathrm{G})$. He is the Co-Chair of IEEE COMSOC SDN-NFV subCommittee. He is a member of the UN Strategy Council, a Member of the present EU Future Internet Forum, representing the Luxembourg government. He sits on the IPv6 Ready Logo Program Board. He is a Program Coordinator at the University of Luxembourg, contributing to several EU-funded projects on next generation technologies.

E-mail: latif.ladid@uni.lu

Faculty of Science, Technology and Medicine

Department of Computer Science

Université du Luxembourg

Luxembourg 